\title{
La forme suit la fonction
}

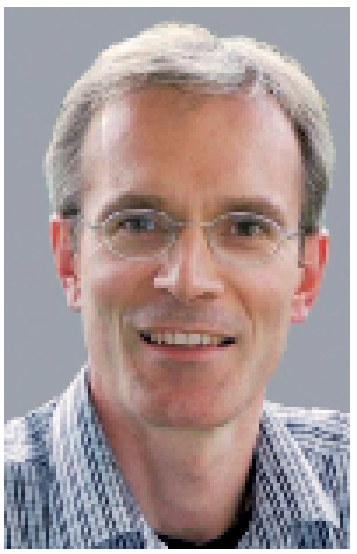

Tempora mutantur, et nos mutamur in illis. Les temps changent, le Bulletin des médecins suisses aussi, pourrait-on dire par analogie à la sentence latine. Vous l'avez sans doute remarqué, chères lectrices et lecteurs, à la vue de l'actuelle édition. Et comme il en est souvent d'un changement qui se produit sans crier gare, vous vous demandez peut-être ce que cette nouvelle présentation signifie. Elle n'est en tout cas pas censée être une fin en soi. On pourrait bien davantage comparer le principe qui nous a guidés en la créant à un axiome de l'architecture moderne: la forme suit la fonction. Le but de notre travail a été de mettre à disposition des formes et des instruments permettant au BMS de remplir ses multiples fonctions de manière optimale:

- Le BMS est l'organe officiel de la FMH. Les informations de la FMH doivent atteindre leur public le plus directement possible. Il nous a semblé ainsi logique de transformer les pages FMH, qui paraissaient jusqu'ici dans la section politique, en rubrique à part entière qui comprenne les prises de position officielles, les communications, etc. de la Fédération des médecins suisses. Vu son importance, cette rubrique occupe la première partie de la revue, qui s'ouvre sur l'éditorial, rédigé en règle générale par un membre du Comité central.

- Les informations officielles d'importantes organisations de la santé publique doivent être clairement discernables d'autres contributions plus libres et spontanées. Deux nouvelles rubriques auront ainsi droit de cité: Organisations de la Chambre médicale et Autres organisations.

- Selon son statut rédactionnel, le BMS a la fonction de tremplin de discussion et d'information, librement accessible au corps médical et à d'autres cercles s'intéressant et coopérant au domaine de la santé. Composées d'articles de l'extérieur ou de la rédaction ellemême, les rubriques Le journal et Culture servent cet objectif.

Comme auparavant, le but principal de la rédaction est d'offrir un forum ouvert à l'information et à la discussion, destiné à tous les membres de la FMH, mais aussi au reste du corps médical et aux milieux liés au monde de la santé. En regard de la diversité des médecins réunis au sein de la FMH, qui se traduit tant sur le plan de la philosophie de la santé et des méthodes employées que des aspects politiques, idéologiques et socioculturels, la tâche est énorme, mais le défi en vaut la peine. Vouloir satisfaire tout le monde serait une gageure équivalent à résoudre la quadrature du cercle, ou à tenter d'enthousiasmer tout à la fois le lectorat de Libération et du Figaro.

$\mathrm{Vu}$ sous cet angle, le Bulletin des médecins suisses pourrait se faire le miroir du paysage médical suisse. Le reflet, sous une forme condensée, de contrastes aigus entre des confrères appelés inopinément à se présenter côte à côte alors que dans la vraie vie, ils s'en garderaient bien. Un exemple est fourni par la présente édition donnant la parole - en allemand uniquement - à deux auteurs de renom et membres de la FMH, Max Geiser et Walter Buschauer, séparés par l'opposition radicale de leurs visions sur la «bonne» et «véritable» médecine.

La Fédération des médecins suisses est une organisation ouverte et pluraliste. Dans la mesure où les auteurs évoluent dans le vaste cadre que s'est donné la FMH elle-même, nous ne nous voyons pas dans le rôle de juge interdisant la parole à l'une des parties. Nous souhaitons bien davantage contribuer, par-delà la rigueur et l'âpreté des débats, à reconnaître l'effort de l'adversaire, à travailler pour le bien du patient et à faire avancer la médecine en tant que science et art de guérir. Vu le climat inhospitalier de la politique sanitaire auquel les médecins de tous bords se voient chaque jour davantage exposés, un progrès serait, à mon avis, de faire preuve d'un esprit prônant les dénominateurs communs. C'est dans ce sens-là que je vous souhaite, à l'avenir également, une bonne lecture.

Bruno Kesseli, rédacteur en chef 